The following pages constitute the final, accepted and revised manuscript of the article.

Nylén, Anders, Larsson, Bengt, Skagerberg, Gunnar

"A sequential fluorescence method for neurotransmitter specific retrograde tracing in the central nervous system of the rat; utilizing True Blue and immunohistochemistry in combination with computer assisted photography."

Brain Res Brain Res Protoc. 2005 May;15(1):30-7.

Publisher: Elsevier.

Use of alternative location to go to the published version of the article requires journal subscription.

Alternative location: http://dx.doi.org/10.1016/j.brainresprot.2005.03.001 


\title{
A sequential fluorescence method for neurotransmitter specific retrograde tracing in the central nervous system of the rat; utilizing True Blue and immunohistochemistry in combination with computer assisted photography
}

\author{
Anders Nylén, Bengt Larsson, Gunnar Skagerberg \\ Departments of Clinical Pharmacology (A.N., B.L.) and Neurosurgery (G.S.) Lund \\ University Hospital S-221 85 Lund, Sweden
}

Total number of pages: $11+5$

Author for correspondence:

Anders Nylén, Ph.D.

Institute of Laboratory Medicine Department of Clinical and Experimental

Pharmacology, Lund University Hospital, Lund University, S-221 85 Lund, Sweden

Phone: +46-46-173351, Fax: +46-46-2111987

e-mail: anders.nylen@klinfarm.lu.se

Abstract 
Aiming to map the distribution of spinally projecting, hypothalamic neurons containing neuronal nitric oxide synthase (nNOS), True Blue (TB) is injected into the rat spinal cord. After survival times of 7 to 14 days the animals are anaesthetized and perfused transcardially with a solution containing paraformaldehyde and sucrose. After dissection, the injection site is further fixed for 4-8 hours, cut in a cryostat and documented by computer assisted digital photography. The brain region of interest is fixed for four hours, rinsed in phosphate buffer for 48 hours, sectioned and photographically documented utilizing filter settings for visualization of TB. The brain sections are then immunohistochemically processed using a primary antibody against nNOS and a Texas Red (TR)-labelled secondary antibody and once again photographically documented, now using filter settings for visualization of TB and TR respectively. Utilizing the Photoshop program, the TB containing cells can then be exactly aligned and the presence of TB and/or TR fluorescence in the same cell bodies are evaluated. This method for neurotransmitter-specific retrograde tracing derives its high sensitivity from the optimization of fixation/rinsing parameters, the use of appropriate fluorophores and sequential digital microphotography.

Theme: Cellular and molecular biology

Topics: Staining, tracing, and imaging techniques

Key words: Digital photography; True Blue; Fluorescence; Retrograde tracer; Spinal cord; Immunohistochemistry

\section{Introduction}

The combination of retrograde tracing and immunohistochemistry allows for mapping of 
axonal projections of cell bodies containing a defined neurotransmitter or transmitterrelated enzyme. Such methods were described already in the mid seventies utilizing conventional light-microscopy and photography. The technique initially relied on the different distribution and morphology of Horse Radish Peroxidase, whether bound to an antibody for visualising the transmitter, or retrogradely transported from a distal injection site in order to demonstrate the neuronal projection [7]. A simpler and apparently more sensitive technique was later provided by the combination of a natively fluorescent retrograde tracer [6] with either fluorescence histochemistry [3] or immunofluorescence [10] utilizing fluorophores with non-overlapping spectral characteristics [5]. A protocol for the latter method was provided by Sawchenko and Swanson [10], who used the fluorescent retrograde tracer True Blue (TB) in combination with immunofluorescence utilizing a fluorescein isothiocyanate (FITC)-labelled secondary antibody. Although these methods were shown to be highly useful, they are somewhat cumbersome to use, mainly because conventional photography is time consuming and sometimes difficult to perform because of insufficient fluorescence intensity of the tracer. Furthermore, the sensitivity of the technique sometimes appears less than optimal probably due to partly overlapping spectral characteristics, diffusion and fading of the retrograde tracer during tissue processing and the consequent need for long photographic exposure times when using conventional photography. These drawbacks are largely overcome by the present protocol, which utilizes a modified procedure for tissue processing in combination with fluorophores with non-overlapping spectral characteristics, and takes advantage of the recent developments for digitalized photography which allows for short exposure times and subsequent, but immediate, computerized image analysis and processing. Utilizing TB in combination with a neuronal nitric oxide synthase (nNOS) antibody, the nitrergic projections originating in the hypothalamic paraventricular nucleus (PVN) was investigated [9]. In this protocol retrogradely transported TB and Texas Red (TR) bound to the secondary antibody are selectively visualized using amino methylcoumarin acetic acid (AMCA) and TR filter settings respectively.

\section{Type of research}

Neuroanatomical studies concerned with the origins, projections and terminations of neuronal systems containing a specified neurotransmitter.

\section{Time required}

Seven to 14 days for retrograde tracing. Approximately $8 \mathrm{~h}$ for preparation of sections and photography session I. Approximately $24 \mathrm{~h}$ for immunohistochemical processing and photography session II. Thus, the complete protocol can be executed in approximately 9 to 16 days.

\section{Materials}

\subsection{Animals}

Female virgin Sprague-Dawley rats weighing 200-300 g (B\&K Universal, Sollentuna, Sweden) are housed, up to four animals together in each cage, under standard laboratory conditions. The rats have free access to water and standard pellets and are housed under controlled conditions at $22 \pm 2{ }^{\circ} \mathrm{C}$ with $12 \mathrm{~h}$ of light and $12 \mathrm{~h}$ of darkness (the experimental protocol was approved by the Animal Ethics Committee, University of 
Lund).

\subsection{Special equipment}

-Camera: SONY DKC-5000, 3CCD digital camera

-Computer: PC, Windows 98 (Compliq, Lund, Sweden)

-Cryostat: Leica CM 3050 (Leica Microsystems Nussloch GmbH, Nußloch, Germany)

-Injection equipment: Hamilton microsyringe $(10 \mu \mathrm{l})$ equipped with bevelled steel cannula, with an outer diameter of 0.47 mm (Hamilton Company, Reno, NV 89502, USA)

-Microscope: Olympus BX 40 (HBO 103W/2 mercury lamp) equipped with objective (Uplan Apo 10x/0.40) and filter settings for AMCA fluorescence (U-MNU, no. 37274, excitation: 360-370 nm, dichroic mirror: $400 \mathrm{~nm}$, barrier filter: $420 \mathrm{~nm}$, injection site and brain area), TR fluorescence (U-MWIY, no. 37273, excitation: 545-580 nm, dichroic mirror: $600 \mathrm{~nm}$, barrier filter: $610 \mathrm{~nm}$, brain area) and FITC fluorescence (U-MWB, no. 37266, excitation: 450-490 nm, dichroic mirror: $500 \mathrm{~nm}$, barrier filter: $515 \mathrm{~nm}$, injection site)

-Operating microscope: Zeiss OPMI-6H (Carl Zeiss, Jena, Germany)

-Soft ware: Image Access (Digital Arts and Science Corporation, Alameda, CA, USA) for image analysis, PC version of Adobe Photoshop ${ }^{\circledR}$ (4.0) for camera control

-Stereotaxic apparatus (Stoelting Co., Wood Dale, IL, USA)

\subsection{Retrograde tracer}

-TB (EMS-POLYLOI GmbH, Groß-Umstadt, Germany)

A $3 \%$ weight/volume (w/v) suspension of TB is prepared by suspending $3 \mu$ g of TB in $0.1 \mathrm{ml}$ distilled water and sonicating for 10 minutes (sonicator; Model B-30, Branson Sonic Power Company, Danbury, CT, USA). This suspension may be stored in a refrigerator for at least 6 months without appreciable loss of fluorescence intensity.

\subsection{Antibodies}

-Rabbit nNOS antiserum (code no. 9224, Euro-Diagnostica, Malmö, Sweden)

-TR-conjugated $\mathrm{F}(\mathrm{ab})_{2}$ fragment donkey anti-rabbit IgG (1:80; code 711-076-152, Jackson ImmunoResearch Inc, West Grove, PA, USA)

\subsection{Chemicals}

-Etylene Glycol-bis (b-aminoethylether)-N,N'N'Tetra Acetic acid (EGTA; Merck KGaA, Darmstadt, Germany)

-Gelatin (Kebo, Stockholm, Sweden)

-Glacial acetic acid (17,4M; Merck Eurolab, Darmstadt, Germany)

-Glycerol (Merck)

-HCl (Merck)

-Heparin, (Lövens, Malmö, Sweden)

$-\mathrm{KCr}\left(\mathrm{SO}_{4}\right)_{2} \mathrm{X} 12 \mathrm{H}_{2} \mathrm{O}$ (Merck)

-KCl (Merck)

-Ketamine, Ketalar ${ }^{\mathrm{R}}$ (Parke Davis, Barcelona, Spain)

$-\mathrm{KH}_{2} \mathrm{PO}_{4}$ (Merck)

-Isopentane (2-methyl butane; BDH laboratories, Poole, England) 
$-\mathrm{MgCl}_{2} \mathrm{x} 6 \mathrm{H}_{2} \mathrm{O}$ (Merck)
$-\mathrm{NaCl}^{\mathrm{Merck})}$
$-\mathrm{NaHCO}_{3}$ (Merck)
$-\mathrm{NaH}_{2} \mathrm{PO}_{4} \mathrm{xH}_{2} \mathrm{O}$ (Merck)
$-\mathrm{Na}_{2} \mathrm{HPO}_{4} \mathrm{x}_{2} \mathrm{H}_{2} \mathrm{O}$ (Merck)
- $\mathrm{NaOH}(\mathrm{Merck}$ )
-Paraformaldehyde (Merck)
-Sucrose (Danisco A/S, Copenhagen, Denmark)
-Triton X-100 (Merck)
-Xylazine; Rompun vet ${ }^{\mathrm{R}}$ (Bayer, Leverkusen, Germany)

\subsection{Chrome alum coated slides}

Dissolve $1 \mathrm{~g}$ gelatin and $0.1 \mathrm{~g} \mathrm{KCr}\left(\mathrm{SO}_{4}\right)_{2} \mathrm{x} 12 \mathrm{H}_{2} \mathrm{O}$ in $200 \mathrm{ml}$ distilled $\mathrm{H}_{2} 0$ at $75^{\circ} \mathrm{C}$ under continuous stirring. Immerse the slides in the filtered solution and let them dry at $60^{\circ} \mathrm{C}$, overnight.

\subsection{Solutions}

-Ca ${ }^{2+}$-free Krebs (CafKr): $1000 \mathrm{ml}$ is prepared as follows: $6.954 \mathrm{~g} \mathrm{NaCl}, 1.26 \mathrm{~g} \mathrm{NaHCO}_{3}$, $0.343 \mathrm{~g} \mathrm{KCl}, 0.244 \mathrm{mg} \mathrm{MgCl}_{2} \times 6 \mathrm{H}_{2} \mathrm{O}$ and $0.1656 \mathrm{~g} \mathrm{NaH} \mathrm{NO}_{4} \mathrm{xH}_{2} \mathrm{O}$ is dissolved in $950 \mathrm{ml}$ distilled $\mathrm{H}_{2} \mathrm{O}$. Make $1000 \mathrm{ml}$ of $0.01 \mathrm{M}$ EGTA by adding $5 \mathrm{ml}$ of $1 \mathrm{M} \mathrm{NaOH}$ to $900 \mathrm{ml}$ distilled $\mathrm{H}_{2} \mathrm{O}$. Add $3.80 \mathrm{~g}$ of EGTA and adjust $\mathrm{pH}$ to 7.4 with glacial acetic acid and dilute with distilled $\mathrm{H}_{2} \mathrm{O}$ to $1000 \mathrm{ml}$ containing heparin (10000 IE/l) and sodium nitrite (2 $\mathrm{g} / \mathrm{l}$ ). Add $10 \mathrm{ml}$ of this stock solution to the above solution and dilute with distilled $\mathrm{H}_{2} \mathrm{O}$ to $1000 \mathrm{ml}$.

$-1000 \mathrm{ml}$ PBS containing $4 \%$ formaldehyde and $15 \%$ sucrose (FAsPBS) is prepared as follows: Dissolve 40 g paraformaldehyde in $64 \mathrm{ml}$ distilled $\mathrm{H}_{2} \mathrm{O}$ at $70^{\circ} \mathrm{C}$ during continous stirring. Add 30 drops of $1 \mathrm{M} \mathrm{NaOH}$ with a pasteur pipette. Keep stirring until the solution is clear. Add $900 \mathrm{ml}$ PBS (recipe as below). Adjust pH to 7.4 with $1 \mathrm{M}$ $\mathrm{NaOH}$. Dissolve $150 \mathrm{~g}$ of sucrose in the solution to reach a final volume of $1000 \mathrm{ml}$. -PBS buffer (PBS): Make $1000 \mathrm{ml}$ solutions with distilled $\mathrm{H}_{2} \mathrm{O}$ of the following: $\mathrm{KH}_{2} \mathrm{PO}_{4} 9.073 \mathrm{~g} / \mathrm{l}$ (solution A), $\mathrm{Na}_{2} \mathrm{HPO}_{4} \times 2 \mathrm{H}_{2} \mathrm{O} 11.866 \mathrm{~g} / \mathrm{l}$ (solution B), $\mathrm{NaCl} 45 \mathrm{~g} / \mathrm{l}$ (solution C). To make $1000 \mathrm{ml}$ PBS take $60 \mathrm{ml}$ of solution A, $140 \mathrm{ml}$ of solution B and $800 \mathrm{ml}$ of solution C. Adjust $\mathrm{pH}$ to 7.2 with $1 \mathrm{M} \mathrm{NaOH}$ or $1 \mathrm{M} \mathrm{HCl}$.

-100 ml PBS/glycerol containing 1,4-phenylene diamine (PBSgp) is prepared as follows (during cold and dark conditions): $100 \mathrm{mg}$ 1,4-phenylenediamine (not in lumps) is diluted in $10 \mathrm{ml}$ PBS $\left(+4^{\circ} \mathrm{C}\right)$ during stirring and use of a glass rod. Add $90 \mathrm{ml}$ glycerol $\left(+4^{\circ} \mathrm{C}\right)$. Store in darkness at $-20^{\circ} \mathrm{C}$.

$-1000 \mathrm{ml}$ PBS containing 15\% sucrose (sPBS) is prepared as follows: Dissolve $150 \mathrm{~g}$ sucrose in PBS to reach a volume of $1000 \mathrm{ml}$.

$-1000 \mathrm{ml} \mathrm{0.2 \%} \mathrm{Triton} \mathrm{in} \mathrm{PBS} \mathrm{(TrPBS)} \mathrm{is} \mathrm{prepared} \mathrm{as} \mathrm{follows:} \mathrm{Dissolve} 2 \mathrm{ml}$ Triton X100 in PBS and dilute to $1000 \mathrm{ml}$.

Purchased antibodies were prepared as follows: Freeze dried primary antibody (50 $\mu$ l) was diluted with $50 \mu \mathrm{l}$ distilled $\mathrm{H}_{2} \mathrm{O}$ and then diluted with $950 \mu \mathrm{l}$ PBS to get a concentration of $1: 20$ and then divided into aliquots and frozen at $-20^{\circ} \mathrm{C}$. Freeze dried secondary antibody $(0.3 \mathrm{mg})$ was first diluted with $250 \mu \mathrm{l}$ distilled $\mathrm{H}_{2} \mathrm{O}$ and thereafter 
with $250 \mu \mathrm{l}$ of glycerol, to get a concentration of 1:2 and then divided into aliquots and frozen at $-20^{\circ} \mathrm{C}$.

\section{Detailed procedure}

\subsection{Tracer injection}

\subsubsection{Anaesthesia and surgical exposure}

During the light period, the animals are anaesthetized with a mixture of ketamine (50 $\mathrm{mg} / \mathrm{kg}$ ) and xylazine (10 mg/kg), administered intramuscularly.

\subsubsection{Surgery}

The animals are secured in a stereotaxic frame with the tooth bar fixed as high as possible. For injection into the spinal cord, a moderate weight is attached to the tail of the animal in order to lift the body so that the thorax can freely expand downwards with inspiration, thereby minimizing movement of the back, so that the spinal cord is almost totally immobilized. The spinal cord is exposed through a midline skin incision followed by stripping the longitudinal muscles, from one or two vertebrae and making a small laminectomy.

\subsubsection{Tracer injection}

Under surveillance through the operating microscope the dura is incised longitudinally, whereafter 0.2-0.8 $\mu \mathrm{l}$ of the TB suspension (see Section 4.3) is injected into the spinal cord via a $10 \mu \mathrm{l}$ Hamilton Syringe during 1-2 minutes. The syringe is left in place for 5 minutes before being slowly withdrawn. Any sign of leakage of tracer observed through the microscope are washed with $0.9 \%$ saline and removed with a piece of surgical sponge. The vertebral muscles are approximated by 4.0 sutures and the skin incision sealed by stapling. The animals are then permitted to wake up before they are again caged (and allowed to survive for 7-14 days).

\subsection{Preparation of tissue}

After 7-14 days, the animals are anaesthetized as in Section 5.1.1. The rats are then perfused transcardially through the ascending aorta, first with $100 \mathrm{ml}$ of $\mathrm{CafKr}$ at $+4^{\circ} \mathrm{C}$, followed by fixation with $250 \mathrm{ml}$ of FAsPBS at $+4^{\circ} \mathrm{C}$ (see Section 4.5). The parts of the spinal cord which contain the TB injection-site are dissected and immersion-fixed in FAsPBS for 4 to $8 \mathrm{~h}$ and directly frozen in isopentane $\left(-40^{\circ} \mathrm{C}\right)$ until the end of "boiling" and then stored at $-70^{\circ} \mathrm{C}$. When convenient, serial cryostat sections are cut at $-20^{\circ} \mathrm{C}$, perpendicularly to the longitudinal axis of the spinal cord at a thickness of $10 \mu \mathrm{m}$. Following perfusion fixation, the brains are dissected out. Small identifying cuts are made in the cortex ipsilaterally to the injection site in frontal slices, which are about 7 $\mathrm{mm}$ thick, containing the region of interest. The slices are immersion-fixed in FAsPBS at $+4^{\circ} \mathrm{C}$ for $4 \mathrm{~h}$ and rinsed in sPBS at $+4^{\circ} \mathrm{C}$ for $48 \mathrm{~h}$ (three rinses), frozen and stored as above. Serial cryostat sections are cut in the frontal plane at a thickness of $10 \mu \mathrm{m}$ at $20^{\circ} \mathrm{C}$. Relevant sections are thaw-mounted onto chrome alum coated slides, and processed as below (Section 5.3-5.5).

\subsection{Photography session I}

Using a microscope connected to a digital camera and equipped with appropriate filter settings (Section 4.2), each injection site is examined and photographed using AMCA 
and FITC filters (Fig. 1). The FITC filter setting is used for delineating the most intense zone of the injection site (zone I; Fig. 1) [11].

Only animals without signs of leakage and meningeal accumulation of TB are used. TB fluorescence is examined and photographed in the relevant brain areas (Fig. 2A).

\subsection{Immunohistochemical processing}

All incubations below, are performed in moisture chambers at room temperature if not otherwise stated. After photography session I, the sections to be examined for retrograde labelling are first immersed in TrPBS for $2 \mathrm{~h}$, and subsequently with rabbit nNOS antiserum $(1: 980 ;[1,2,8,9])$ at $+4^{\circ} \mathrm{C}$ overnight. The following day the sections are rinsed in PBS (3x3 min) and further incubated with TR-conjugated donkey anti-rabbit affinity purified $\mathrm{F}(\mathrm{ab})_{2}$ fragments, dissolved in TrPBS, for $90 \mathrm{~min}$. Finally, the sections are rinsed in PBS (3x3 min) and mounted in PBSgp (Section 4.5). The sections are either handled directly according to Section 5.5 (see below) or stored at $-20^{\circ} \mathrm{C}$ and subsequently handled according to Section 5.5 when convenient (see below).

\subsection{Photography session II}

After the immunohistochemical processing, the brain structures documented in photography session I are again photographed. Both nNOS-TR immunofluorescence and the TB fluorescence which remains after the immunohistochemical processing are documented in the computer using appropriate filter settings. The pattern of the remaining TB fluorescence may be used as template to facilitate alignment, with the corresponding image from photography session I, obtained before immunohistochemical processing.

\subsection{Image processing and evaluation}

In the majority of cases stored images from photography session I and II are processed using a two layer technique in Adobe Photoshop (in rare cases three layers are used). The image of TB fluorescence obtained in photography session I is used as layer one. The colour image of immunoreactivity (TR fluorescence) is converted to greyscale and thereafter inverted, thus showing immunoreactive neurons black on a white background. This image is used as layer two on top of the TB image obtained in photography session I. By using the opacity regulator in the Photoshop program, it is possible to make layer two more or less transparent (Fig. 3). In almost every case there is a miss-match in alignment, between layer one and layer two, due to the difficulties of photographing exactly the same field of view in photography session I and II. When the direction of the miss-match has been visually established, layer two is temporarily removed, and layer one is rotated in order to minimize the miss-match (Fig. 3). Finally, layer two is reintroduced, and horisontally and vertically matched with layer one. By repeating this procedure, a perfect match of layer one and layer two is obtained. From this match a double image can be constructed simultaneously visualising TB fluorescence and immunoreactivity in exactly the same field of view (Fig. 3). Thus, in every structure documented in this way, co-localization and non co-localization of TB fluorescence and immunoreactivity can, within the same neuronal structures, be definitely established.

\section{Results}


As shown in figure 2 the appropriate filter setting reveals either the fluorescent tracer as a light blue, mainly cytoplasmatic light blue fluorescence against a dark back-ground or, alternatively, the distribution of the TR-labelled secondary antibody in cell bodies and fibres as a bright-red fluorescence against a dark-red background. Trying to optimize the possibility to distinguish between TR and TB, the image of immunoreactivity (layer 2) was first converted to greyscale and then inverted in Photoshop ${ }^{\circledR}$ creating a picture exhibiting dark neurons on a light background (see Fig. 3). By comparing the appearance of labelling within the two layers at different degrees of transparency (by using the opacity regulator; Fig. 3) the absence or presence of either type of fluorescence ("retrograde" or "immunoreactive") can readily be assessed within any chosen area of interest.

Neuronal cell bodies were observed that contained both TB fluorescence and nNOSimmunoreactivity, together with cell bodies that contained either TB fluorescence or nNOS-immunoreactivity (see Fig. 2). A marked reduction of TB fluorescence was found when comparing the sections containing $\mathrm{TB}$ at the region of interest before and after immunohistochemical processing (Fig. 4).

\section{Discussion}

In comparison with previous methods the present protocol derives its superior sensitivity and convenience from three main factors, which together with some comments relating to the delineation of the injection site will be discussed in paragraphs below.

\subsection{Injection site}

Although the relation between the appearance of the injection site and the area of efficient uptake of tracer is difficult to determine, it seems very important to try to visualise the injection site and if possible to describe it in at least semi-quantitative terms. In this protocol we have chosen to visualise the injection site using two different filter settings providing both optimal and clearly suboptimal excitation of TB fluorescence (using the AMCA and FITC filter settings, respectively) in order to quantitatively delineate three different zones of the injection site (Fig. 1) [11]. By keeping the tissue that contains the injection site, in sucrose-containing fixative until freezing and sectioning, we have aimed at minimizing postmortem diffusion of the tracer.

\subsection{Tissue handling}

By adding sucrose to the fixative and keeping the tissue in this fixative until sectioning post-mortem reduction of the tracer intensity is minimized until its distribution has been documented in photography-session I. Although some tracer fluorescence remains after the immunohistochemical processing there appears to be a marked wash-out of TB during this step, usually leaving enough TB to allow for orientation, though clearly not sufficient for anatomical mapping purposes (Fig. 4). While we did not found the present procedure including mounting and cover-slipping to cause troublesome shrinkage or elastic changes, the protocol may well be applied as an on-slide method if such morphological artefacts represents a concern.

\subsection{Spectral characteristics}


As shown in figure 5 both the excitation and emission spectra for TB and TR differ widely with virtually no overlap allowing for selective visualization of either fluorochrome $[3,4]$. This provides a clear-cut advantage in comparison with FITC whose spectral characteristics lie in between a partly overlap with both TB and TR. In this study an AMCA filter is used to detect TB.

\subsection{Computerized photography and image handling}

The use of light sensitive digital cameras has two major advantages: First, they drastically reduce the required exposure time and accordingly the fading of fluorescence. Second, digital cameras allow a direct and repeated documentation, if required. In rare cases the TB image from photography session II may be used as a third layer to facilitate orientation.

\section{Quick procedure}

Tracer injection

10-14 days survival

Perfusion and subsequent immersionfixation.

Cryostat sectioning

Photographic session I

Immunohistochemical processing

Photographic session II

\section{Acknowledgements}

This study was performed during 1998-2000 at the department of pathology in Lund, Lund University, Sweden. We are most grateful to Professor Per Alm and Doctor Bo Holmqvist for valuable instructions and criticism, and for the use of the equipment, which was a pre-requisite for the accomplishment of the study. The excellent technical assistance of Mrs Lillemor Thuresson, Department of Pathology, Lund University Hospital, is gratefully acknowledged. This work was supported by the Swedish Medical Research Council (grants no 6837 and 11205), the Medical Faculty of Lund University, the foundations of Magnus Bergwall, Crafoord, Anna Lisa and Sven-Eric Lundgren for medical research, Åke Wiberg and Thelma Zoëga, and the Royal Physiographic Society, Lund, Sweden.

\section{Essential literature references}

\section{$[9,10]$}

\section{References}

[1] Alm P, Larsson B, Ekblad E, Sundler F, Andersson K-E. Immunohistochemical localization of peripheral nitric oxide synthase-containing nerves using antibodies raised against synthesized $\mathrm{C}$ - and $\mathrm{N}$-terminal fragments of a cloned enzyme from rat brain. Acta Physiol. Scand. 148 (1993) 421-429.

[2] Alm P, Skagerberg G, Nylén A, Larsson B, Andersson K-E. Nitric oxide synthase and vasopressin in rat circumventricular organs. An immunohistochemical study. Exp. Brain Res. 117 (1997) 59-66. 
[3] Björklund A, Skagerberg G. Simultaneous use of retrograde fluorescent tracers and fluorescence histochemistry for convenient and precise mapping of monoaminergic projections and collateral arrangements in the CNS. J. Neurosci. Methods (1979) 261277.

[4] Haugland RP. Handbook of fluorescent probes and research products, ninth edition (2002) Molecular probes, Eugene, Oregon, USA.

[5] Hökfelt T, Skagerberg G, Skirboll L, Björklund A. Combination of retrograde tracing and neurotransmitter histochemistry. In: A. Björklund, T. Hökfelt (Eds.), Handbook of Chemical Neuroanatomy, Vol. 1: Methods in Chemical Neuroanatomy, Elsevier Science Publishers B.V., Amsterdam, 1983, pp 228-285.

[6] Kuypers HG, Bentivoglio M, van der Kooy D, Catsman-Berrevoets CE. Retrograde transport of bisbenzimide and propidium iodide through axons to their parent cell bodies. Neurosci Lett. 12 (1979) 1-7.

[7] Ljungdahl Å, Hökfelt T, Goldstein M, Park D. Retrograde peroxidase tracing of neurons combined with transmitter histochemistry. Brain Res. 84 (1975) 313-319.

[8] Nylén A, Skagerberg G, Alm P, Larsson B, Holmqvist BI, Andersson K-E. Detailed organization of nitric oxide synthase, vasopressin and oxytocin immunoreactive cell bodies in the supraoptic nucleus of the female rat. Anat. Embryol. (Berl). 203 (2001) 309321.

[9] Nylén A, Skagerberg G, Alm P, Larsson B, Holmqvist BI, Andersson K-E. Nitric oxide synthase in the hypothalamic paraventricular nucleus of the female rat; organization of spinal projections and coexistence with oxytocin or vasopressin. Brain Res. 908 (2001) 10-24.

[10] Sawchenko PE, Swanson LW. A method for tracing biochemically defined pathways in the central nervous system using combined fluorescence retrograde transport and immunohistochemical techniques. Brain Res. 210 (1981) 31-51.

[11] Skagerberg G, Björklund A, Lindvall O. Further studies on the use of the fluorescent retrograde tracer True Blue in combination with monoamine histochemistry. J. Neurosci. Methods. 14 (1985) 25-40.

\section{Figures}

Fig. 1. Transverse section through the spinal cord injection site, delineating the different injection zones [11]. A: AMCA filter setting preferentially visualizing TB fluorescence. $B$ : The same structure visualized using FITC filter setting, which reveals the most 
intensive zone of the injection site (zone I), and further outlines the grey matter. C: Schematic illustration, compiled from several adjacent sections, where injection site zones I (black), II (dark grey) and III (light grey) are shown [11]. Scale bar represents 50 $\mu \mathrm{m}$.

Fig. 2. Frontal section through the PVN. TB-labelled cell bodies (A) and nNOS-IR cell bodies (B). Thick, short arrows indicate cell bodies exhibiting both TB-labelling (AMCA filter) and nNOS-immunoreactivity (TR filter). Thin, long arrows indicate cell bodies exhibiting either TB-labelling or nNOS-immunoreactivity. 3V indicates third ventricle. Scale bar represents $50 \mu \mathrm{m}$.

Fig. 3. An example of the use of two layers when constructing an image for mapping both a retrograde tracer and an antigen, visualized by TB (layer one) and TR-fluorescence (layer two) respectively. A: 0\% opacity of layer two to layer one. B: 25\% opacity. C: 50\% opacity. D: $75 \%$ opacity. E: 100\% opacity. The arrow in A-E indicates a neuron exhibiting both TB-labelling, and in this case, nNOS-immunoreactivity. The nNOS image was inverted compared to what originally observed (see Fig. 2). This was performed to establish a clear contrast between image layers one and two (see Section 5.6). Scale bar represents $50 \mu \mathrm{m}$.

Fig. 4. PVN. A marked reduction of TB fluorescence was found after immunohistochemical processing (B) compared to before immunohistochemical processing (A). $3 \mathrm{~V}$ indicates third ventricle. Scale bar represents $50 \mu \mathrm{m}$.

Fig. 5. A: Excitation spectra for TB, FITC and TR. B: Emission spectra for TB, FITC and TR. The value on the $\mathrm{X}$-axis represents wavelength. The value on the $\mathrm{Y}$-axis represents relative excitation (A) and relative emission (B). Data from $[3,4,5]$. 

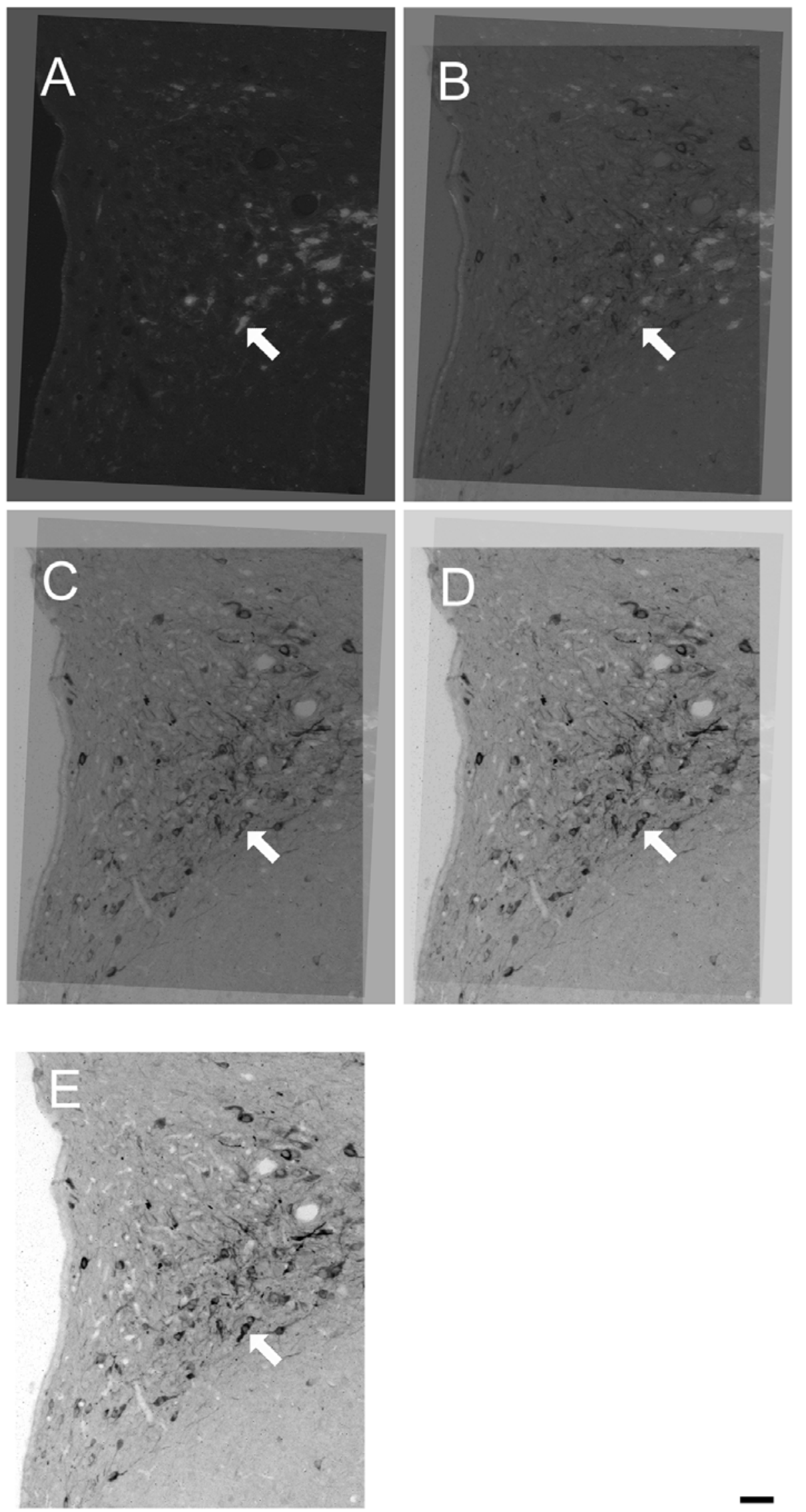
Excitation
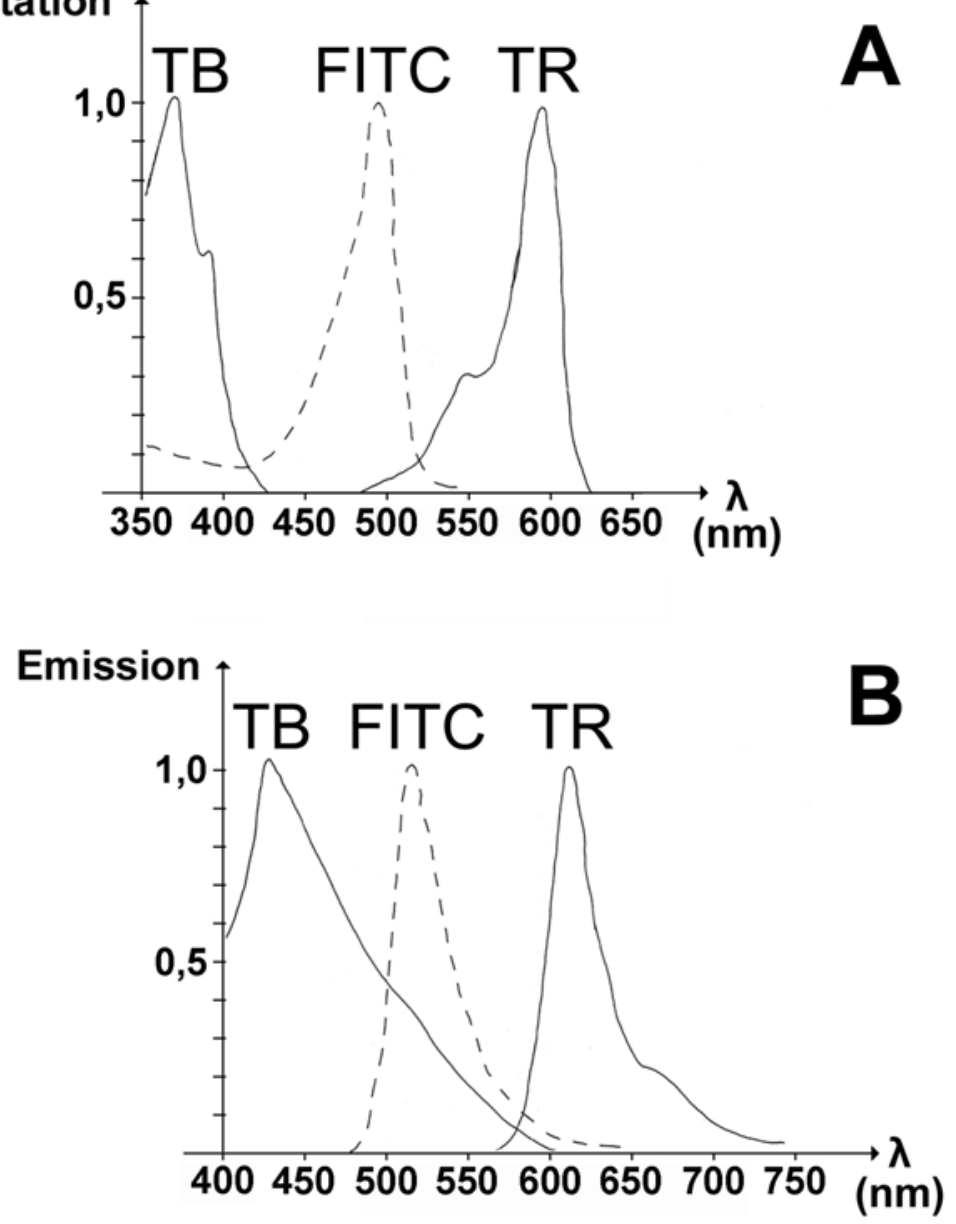


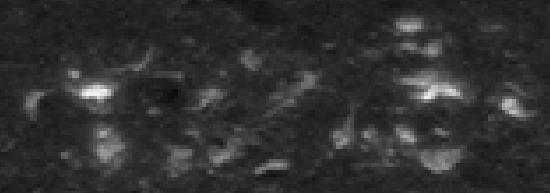

$3 \mathrm{~V}$

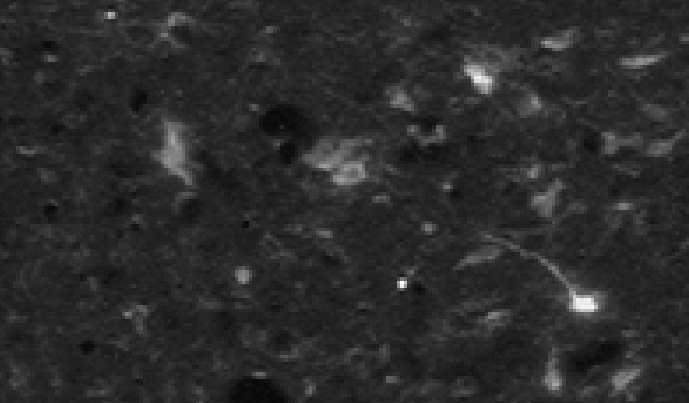

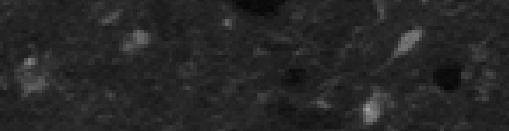

\section{B}

